\title{
Molecular point-of-care testing for influenza to improve early neuraminidase inhibitor treatment and outcomes in hospitalised adults
}

Nathan J Brendish ${ }^{1 *}$, Tristan W Clark ${ }^{1,2,3,4}$

1. Academic Unit of Clinical and Experimental Sciences, Faculty of Medicine, University of Southampton, Southampton, UK.

2. Department of Infection, University Hospital Southampton NHS Foundation Trust, Southampton, UK.

3. NIHR Southampton Biomedical Research Centre, University Hospital Southampton NHS Foundation Trust, Southampton, UK.

4. NIHR Post-Doctoral Fellowship Programme, UK.

* Corresponding author: Dr Nathan J Brendish

LB25, South Academic Block, Department of Infection, Southampton General Hospital, University Hospital Southampton NHS Foundation Trust, Tremona Road, Southampton, SO16 6YD, United Kingdom. $\quad$ nathan.brendish@uhs.nhs.uk Tel: 0044 (0)2381206767, Fax: 0044 (0)2381205023 Alternate author: Dr Tristan W Clark

LF101, South Academic Block, University of Southampton, Southampton General Hospital, University Hospital Southampton NHS Foundation Trust, Tremona Road, Southampton, SO16 6YD, United Kingdom. t.w.clark@soton.ac.uk Tel: 0044 (0)2381208410 Fax: 0044 (0)2381205023 Keywords: point-of-care test, influenza, oseltamivir, neuraminidase inhibitors, hospitalization Running title (max 40 characters including spaces): Molecular point-of-care testing for flu 


\section{TO THE EDITOR -}

We read with great interest the findings by Katzen et al. suggesting that neuraminidase inhibitor (NAI) treatment of influenza infection in hospitalised adults, administered early ( $<6$ hours) after admission, is associated with reduced length of hospital stay and mortality [1]. This is the first study to evaluate the effect of timing of NAI use from admission rather than from onset of symptoms and is highly relevant to clinical practice as most patient with seasonal influenza present after 48 hours of symptoms duration - the time period during which NAI use is thought to be most effective. The effect of timing, from admission to administration, on NAl effects is scientifically credible and mirrors the finding in antibiotic treatment of severe bacterial infection which has led to widespread use of early antibiotic therapy for suspected sepsis. The authors suggest that in line with national guidelines $[2,3]$ promoting the early liberal use of empirical NAI (i.e. used presumptively prior to the results of laboratory testing) in patients with suspected influenza, can achieve this goal.

However, as their study demonstrates, adherence to guidelines advocating empirical NAI use is suboptimal and NAls are often withheld in patients with suspected influenza. In addition, clinician judgement has been shown to be poor at discriminating which patients have influenza and which patients do not (even during periods of peak transmission) thereby missing treatment opportunities in patients with influenza, and causing unintended NAI exposure in those without [4]. Concerns have been raised about unnecessary patient exposure to NAI treatment in patients who do not have influenza virus infection because of adverse events including nausea and vomiting [5]. Therefore a strategy enabling early pathogen-directed therapy, rather than relying on undirected empirical treatment, is clearly desirable.

New molecular test platforms that generate rapid results, are easy to use, have accuracy comparable to laboratory PCR, and can be deployed as point-of-care tests (POCT) for influenza in hospitals, could achieve this aim [6]. In our pragmatic, randomised controlled trial of routine molecular POCT for respiratory viruses, versus standard care, in adults presenting to hospital with acute respiratory illness [7] we showed a median turnaround time for result of 1.6 hours with POCT compared to around 30 hours for laboratory RT-PCR $(p<0.0001)$. In the POCT group $82 \%$ of all NAI use occurred in influenza positive patient versus $47 \%$ in the control group $(p=0.0001)$ and the median number of doses of oseltamivir in those patients who received an NAI but did not have influenza was 1 doses in the POCT group compared with 5 doses ( 2.5 days) in the control group ( $p=0.0003$ ) [7]. In addition 91\% of influenza-positive patients in the POCT group received a NAI compared with only $65 \%$ in the control group ( $p=0.0026$ ). POCT was also associated with higher rates of early hospital discharge and 
early discontinuation of antibiotics and post hoc analysis demonstrated that this was optimized with a turnaround time of $<1.6$ hours, compared with longer turnaround times. [8]

The study by Katzen et al. reinforces the importance of early NAI use in hospitalised adults with influenza to improve patient outcomes. Our work shows that this can be achieved with a routine molecular POCT strategy, which unlike the current paradigm of empirical NAI use, use allows early pathogen-directed therapy and avoids unnecessary drug exposure in influenza negative patients. If further studies confirm these clinical benefits and demonstrate the cost effectiveness of this strategy, molecular POC testing for influenza should replace empirical NAI use in hospitals.

\section{Disclaimers}

The views expressed in the submitted article are the authors' own and not an official position of the institutions to which they belong.

\section{Acknowledgments}

We would like to acknowledge the patient-participants, staff and research institutions in University Hospital Southampton NHS Foundation Trust who contributed to the ResPOC trial.

\section{Funding}

This work was supported by no specific funding. The ResPOC trial was funded by the University of Southampton, with support from the Clinical Research Network Wessex, NIHR Southampton Clinical Research Facility and NIHR Southampton Biomedical Research Centre.

\section{Financial support}

TWC is the holder of an NIHR Post-Doctoral Fellowship [PDF 2016-09-061]. The views expressed in this publication are those of the authors and not necessarily those of the NHS, the National Institute of Health Research, or the Department of Health.

\section{Potential conflicts of interest}

TWC has performed paid consultancy work for Janssen, Roche diagnostics, Synairgen Research Limited and Planet Innovation. He has received speaker fees and travel fees from BioFire Diagnostics, LLC and BioMeriuex. He has received discounted equipment and consumables for the purposes of research from BioFire Diagnostics, LLC. He has acted as chief or principal investigator in clinical trials of antivirals sponsored by Gilead Sciences and Janssen. He has enrolled patients into clinical trials sponsored by Novartis, GlaxoSmithKline and Baxter Pharmaceuticals. NJB has recruited patients into clinical trials sponsored by Janssen and Gilead Sciences.

\section{References}


1. Katzen J, Kohn R, Houk JL, Ison MG. Early oseltamivir after hospital admission is associated with shortened hospitalization: A five-year analysis of oseltamivir timing and clinical outcomes. Clin Infect Dis 2018. [Epub ahead of print].

2. Centers for Disease Control and Prevention. Influenza guidelines. https://www.cdc.gov/flu/professionals/antivirals/summary-clinicians.htm. [Last accessed 24th October 2018].

3. Public Health England. PHE guidance on use of antiviral agents for the treatment and prophylaxis of seasonal influenza. Version 8.0, September 2017. https://www.gov.uk/government/publications/influenza-treatment-and-prophylaxis-usinganti-viral-agents. [Last accessed 24th October 2018].

4. Dugas AF, Valsamakis A, Atreya MR, et al. Am J Emerg Med 2015;33(6):770-5.

5. Kelly H, Cowling BJ. Influenza: the rational use of oseltamivir. Lancet 2015;385(9979):17001702.

6. Brendish NJ, Schiff HF, Clark TW. Point-of-care testing for respiratory viruses in adults: The current landscape and future potential. J Infect 2015;71(5):501-10.

7. Brendish NJ, Malachira AK, Armstrong $L$, et al. Routine molecular point-of-care testing for respiratory viruses in adults presenting to hospital with acute respiratory illness (ResPOC): a pragmatic, open-label, randomised controlled trial. Lancet Respir Med 2017;5(5):401-411.

8. Brendish NJ, Malachira AK, Beard KR, et al. Impact of turnaround time on outcome with point-of-care testing for respiratory viruses: a post hoc analysis from a randomised controlled trial. Eur Respir J 2018;52(2). pii: 1800555. 